\title{
Las competencias en la digitalización: experimento en el entorno de un curso internacional \\ Competencies in digitalization: An experiment in an international course
}

\author{
Amaya Erro-Garcés ${ }^{1}$, Celia Hernández Palaceto ${ }^{2}$ \\ amaya.erro@unavarra.es, celiahernandez@uv.mx
}

\author{
${ }^{1}$ Gestión de Empresas \\ Universidad Pública de Navarra (UPNA) \\ Navarra, España
}

\author{
${ }^{2}$ Pedagogía \\ Universidad de Veracruzana \\ Veracruz, México
}

\begin{abstract}
Resumen- La transformación digital afecta a la localización del talento en un entorno donde el cambio digital se vuelve cada vez más rápido y profundo. En este contexto, las competencias digitales son imprescindibles. El objetivo de este trabajo de investigación consiste en analizar las competencias en un entorno digital global a partir de un experimento internacional desarrollado conjuntamente por la Universidad de Veracruz y la Universidad Pública de Navarra. En concreto, se analizan los resultados de tres grupos de estudiantes de ambas universidades que participaron en un curso semipresencial a través de la plataforma Moodle. El contenido del curso se centraba en la Industria 4.0 y la transformación digital. Los estudiantes trabajaron en grupos internacionales donde se comunicaban virtualmente con herramientas de trabajo colaborativo. Los investigadores buscaban definir así un contexto global de trabajo digital y multicultural. Los resultados muestran que las competencias digitales, aunque necesarias, no son suficientes para desarrollar con éxito un trabajo en este entorno. Adicionalmente, se identificaron las competencias más desarrolladas (toma de decisiones y desarrollo cognitivo), así como las que necesitaban refuerzo (las competencias digitales). Este experimento internacional ha definido un escenario de aprendizaje colaborativo que puede ser implementado a mayor escala en un ámbito internacional.
\end{abstract}

Palabras clave: educación intercultural, aprendizaje cooperative, interdisciplinariedad, aprendizaje semipresencial, competencias, transformación digital

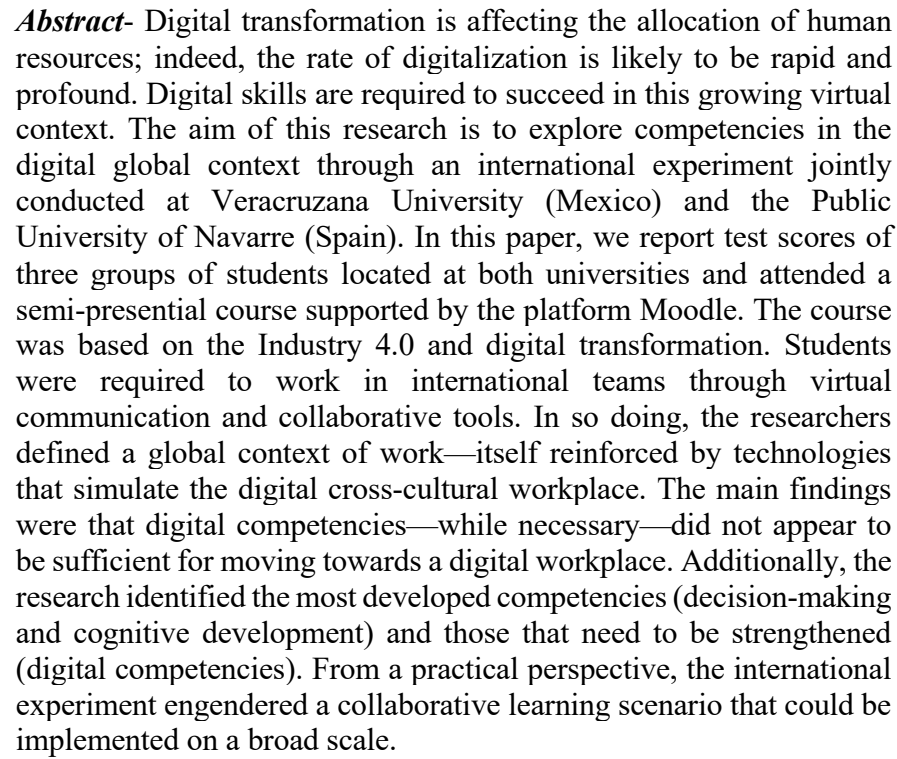

Abstract- Digital transformation is affecting the allocation of human resources; indeed, the rate of digitalization is likely to be rapid and context. The aim of this research is to explore competencies in the digital global context through an international experiment jointly conducted at Veracruzana University (Mexico) and the Public University of Navarre (Spain). In this paper, we report test scores of three groups of students located at both universities and attended a semi-presential course supported by the platform Moodle. The course in international teams through virtual communication and collaborative tools. In so doing, the researchers defined a global context of work-itself reinforced by technologies that simulate the digital cross-cultural workplace. The main findings were that digital competencies - while necessary - did not appear to be sufficient for moving towards a digital workplace. Additionally, the research identified the most developed competencies (decision-making and cognitive development) and those that need to be strengthened implemented on a broad scale.
Keywords: intercultural education, cooperative learning, interdisciplinarity, blended learning; skills, digital transformation

\section{INTRODUCCIÓN}

La transformación digital ha tenido una gran influencia en las rutinas diarias. En particular, las tecnologías han transformado las relaciones humanas, la educación, la comunicación y los hábitos; es probable que estos cambios se vuelvan aún más relevantes en los años siguientes. En resumen, las Tecnologías de la Información y la Comunicación (TICs) han transformado el espacio y el tiempo, complementando el mundo real con uno virtual (Castells, 2010).

Este desarrollo, en general, afecta en mayor medida a los jóvenes. Los jóvenes deben estar preparados para enfrentar este mundo virtual y su desarrollo futuro (Ratten \& Jones, 2020a; Ratten \& Jones, 2020b). Por otro lado, los jóvenes y adolescentes son "nativos" digitales, es decir, habiendo crecido utilizando modalidades digitales, es probable que hayan desarrollado habilidades digitales más altas que las generaciones de mayor edad. Esto les genera una ventaja competitiva sobre otros trabajadores en una amplia gama de profesiones y sectores (De Castro, 2016).

Con este objetivo en mente, muchas instituciones se han centrado en el desarrollo y perfeccionamiento de las habilidades digitales en los jóvenes. De hecho, el Consejo de la Unión Europea considera las competencias digitales como un campo principal de desarrollo del ámbito educativo, y, precisamente, la reforma educativa definida en la Estrategia Europa 2020 incluye las TICs como elemento clave. En la misma línea en el contexto mexicano, la Unión de Empresarios Tecnológicos en Educación (UNETE), una organización sin fines de lucro que tiene como objetivo mejorar la calidad en la educación mexicana, ha estado proporcionando a las escuelas públicas equipos y conectividad; también forma a profesores en competencias de aprendizaje digital. Desde una perspectiva aplicada, hay varias iniciativas para fomentar las habilidades digitales en los jóvenes de Europa y América Latina (por ejemplo, Youth on the Move, An Agenda for New Skills and Jobs, la iniciativa "Opening up Education" para fomentar las habilidades digitales en escuelas y universidades; y los proyectos mexicanos BRAINET, CLASSMATE y KHANACADEMY, entre otros). De hecho, los centros educativos están obligados a adaptar su propuesta para incluir la 
digitalización con el fin de contribuir al desarrollo de estas habilidades informáticas (Picatoste et al., 2018).

Por lo anterior, el presente trabajo de investigación contribuye a comprender el papel de las habilidades digitales para los jóvenes a partir de la definición de un experimento internacional en grupos de universitarios de España y México. Los participantes aprendieron el proceso de digitalización a través de su práctica en un equipo virtual e internacional.

En particular, esta investigación promueve el estudio de las habilidades digitales en los jóvenes mediante (1) la definición de un experimento internacional para formar habilidades digitales que pueden ser replicadas en otros centros educativos, (2) el análisis de los principales resultados derivados del experimento, (3) la identificación de las competencias críticas dentro del contexto digital estudiado, y (4) teniendo en cuenta el papel de la digitalización desde una perspectiva inclusiva (en la que el trabajo en equipo es realizado por herramientas digitales y en equipos virtuales).

\section{DESCRIPCIÓN}

Este estudio muestra el diseño de la intervención estructurada y puesta en marcha de forma colaborativa por la Universidad Pública de Navarra en España y la Universidad Veracruzana en México para promover las habilidades digitales en los estudiantes a través de un curso virtual. El curso se centró en la Industria 4.0 y la transformación digital.

Según ha señalado Touriñán (2011), la intervención educativa es la acción intencionada para llevar a cabo acciones que conduzcan a la consecución del desarrollo integral del alumno. La intervención educativa es de carácter teleológico: hay una asignatura de agente (educador-educador), existe el lenguaje propositivo (se lleva a cabo una acción para lograr algo), se lleva a cabo con el fin de lograr un futuro (el objetivo) y los eventos están vinculados intencionalmente.

Debido a lo anterior, se incorporaron diferentes estrategias didácticas que se utilizaron para llevar a cabo productos académicos que permitieron llegar a los establecidos. Como sugirió Fandos (2003, p. 3), "el desafío actual que tenemos ante las TIC como medio didáctico y su aplicación educativa". El medio nos remite al proceso comunicativo mientras la tecnología se centra en su soporte material. Es tan importante trabajar con el proceso comunicativo que subyace a todo el aprendizaje como estudiar los instrumentos que utilizamos y sus estrategias para ese uso.

Este estudio también tiene un alcance descriptivo. El estudio busca especificar las propiedades de personas, grupos, y comunidades, así como cualquier otro fenómeno que, en algún momento, sea relevante para el análisis (Hernández, Fernández, \& Baptista, 2010).

Como instrumento, se construyó una escala ordinal tipo Likert, a partir de 43 preguntas divididas en 4 categorías delimitadas de acuerdo a la temática que estamos abordando y al trabajo virtual que realizaron los estudiantes 1) Interacción (comunicación, empatía, motivación, adaptación y colaboración se miden en el eje); 2) Desarrollo cognitivo (este eje mide la capacidad de entender, dominio del contenido, síntesis, gestión de la información, logro de objetivos); 3 ) Uso de la plataforma-uso del idioma (en este eje se mide la capacidad de navegar por la plataforma, buscar información, comando de inglés, uso de recursos didácticos); 4) Toma de decisiones (este eje mide la capacidad organizativa, la consecución de objetivos, la claridad en las actividades a realizar, la evaluación de competencias y conocimientos, la distribución de las actividades a realizar, la toma de decisiones como equipo de trabajo). Los valores de escala corresponden a "1", nivel bajo, "2", nivel medio; y "3" relativo al nivel alto. Esta escala está destinada a evaluar qué habilidades digitales desarrollaron los estudiantes durante el curso virtual internacional. Este instrumento fue validado antes de su aplicación por 2 docentes expertos en temas sobre educación y tecnología.

\section{A. Una visión general del experimento}

\section{$1^{a}$ etapa: Análisis del contexto}

El movimiento digital global que estamos experimentando a diario requiere un encuentro constante y permanente con el desarrollo tecnológico en la vida urbana, los negocios, la socialización $\mathrm{y}$, por supuesto, en los ámbitos académico y basado en el conocimiento. Como señaló Rueda (2007, p. 5), a lo largo del desarrollo de la vida humana, los medios de comunicación han jugado un papel importante dentro de él. Estos han contribuido desde el principio a acercar a las personas, en el sentido de que la interacción y la información han sido cada vez más favorecidas. Este hecho se puede ver reflejado patentemente a partir de la invención de la radio, el teléfono, la televisión, el fax, los teléfonos celulares, los satélites, Internet, etc. Estos medios de comunicación, colocándolos en una línea de tiempo, muestran una gran evolución tecnológica que ha permitido cruzar fronteras en una amplia variedad de campos sociales.

Debido a lo que se menciona en el párrafo anterior, la educación superior en sus diversos niveles ha necesitado incorporar cada vez más habilidades y competencias en la gestión de las nuevas tecnologías. Esto permite formar grupos o redes con el fin de la colaboración académica y el intercambio. Las capacidades demostradas por las últimas tecnologías (especialmente las redes) en funciones como la transmisión de información y conocimiento o comunicación han dado lugar a la idea de incorporar estos recursos al propio proceso de formación (Fandos, 2003).

Por estas razones, el curso taller denominado "Competencias en la digitalización: Un experimento en un curso internacional" está diseñado para que los estudiantes incorporen estas herramientas a sus actividades diarias. Esto permitirá a los estudiantes fortalecer sus habilidades para el intercambio académico con la ayuda de plataformas digitales.

La intervención que investigamos surge precisamente de una acción educativa internacional, el curso EMINUS, a través del cual, profesores de varios países colaboraron para diseñar módulos educativos transversales.

\section{$2^{a}$ etapa. Diseño}

Con el fin de aplicar la intervención, se llevó a cabo el diseño instruccional de un curso virtual. Para ello, se siguieron los siguientes pasos:

a. Se llegó a un acuerdo entre la Universidad Pública de Navarra en España y la Universidad Veracruzana en México.

b. Las fechas y horas para llevar a cabo la intervención se definieron de acuerdo con los calendarios de los profesores.

c. Se buscó una plataforma flexible y de fácil acceso, donde los estudiantes pudieran interactuar. 
e. Se definieron temas y subtemas relacionados con el contenido de instrucción 4.0.

f. Las actividades didácticas/pedagógicas fueron diseñadas de acuerdo con cada unidad de contenido (diagramas, mapas mentales, bases de datos, foros, fotografías, etc.).

g. Se establecieron los recursos y medios a utilizar.

h. Se hizo un calendario con las actividades que se llevarán a cabo por semana.

i. Se definieron los criterios de evaluación de cada actividad

j. Por último, se administró una evaluación cuantitativa (aplicación de la escala) a los estudiantes para obtener sus impresiones sobre el curso de taller virtual y las competencias que en mayor o menor media desarrollaron.

El curso taller, "Innovación Tecnológica en el Campo Educativo", fue diseñado para ser impartido en la plataforma virtual titulada "Mis Aulas" (parte de la plataforma Moodle). Se trata de una herramienta digital gratuita que permite la creación de cursos. Se caracteriza por una estructura gratuita que facilita las interacciones profesor-estudiante y estudiante-estudiante.

El curso fue diseñado para desarrollarse en 5 semanas. Es importante subrayar que el requisito era enseñarlo en inglés (con el fin de promover el aprendizaje de otro idioma). Los temas de cada eje están relacionados con la Industria 4.0 y la transformación digital. (Tabla 1)

Tabla 1. Planificación de las actividades

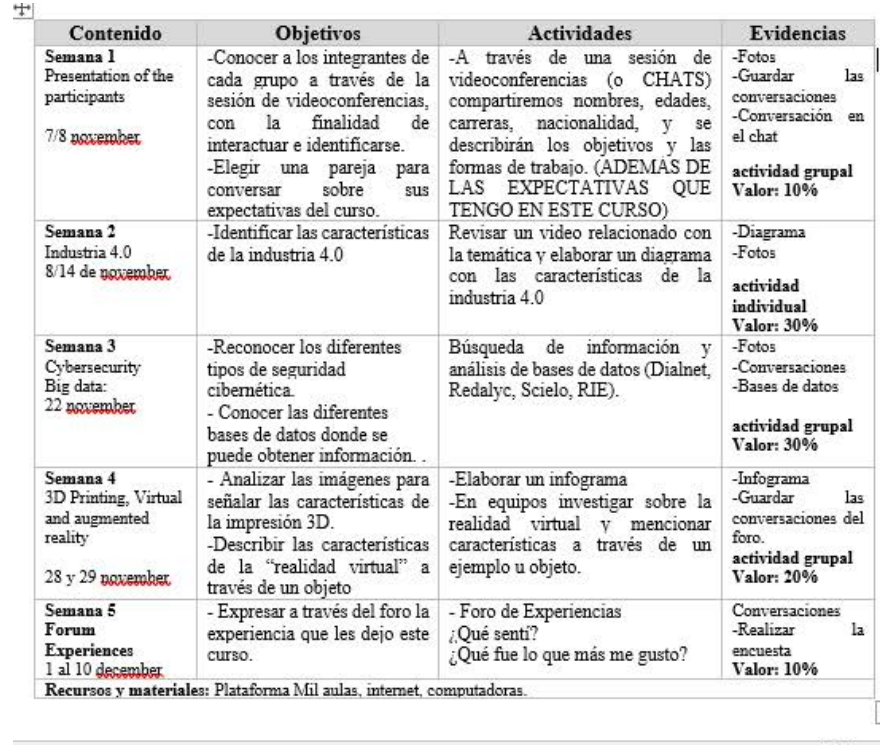

Elaboración propia.

\section{B. Participantes}

Tres grupos de estudiantes $(\mathrm{N}=85)$ participaron en el estudio (Tabla 2). Cincuenta y nueve estudiantes procedían de la Universidad Pública de Navarra. Todos estaban inscritos en la Facultad de Ciencias Económicas y Empresariales; de ellos, 30 estudiantes cursaban estudios para el grado Internacional ADE (21 estudiantes españoles y 9 de otros países). Otro grupo de participantes se pusieron el título internacional de ADE y Economía (22 estudiantes españoles y 7 estudiantes internacionales); 26 asistieron a la Universidad Veracruzana en México, inscrita en el programa de Licenciatura en Pedagogía, con especialización en el Laboratorio de Docencia. La edad de los estudiantes oscilaba entre los 19 y los 26 años (Tabla 2).

\section{Tabla 2. Estructura de los grupos de trabajo}

\begin{tabular}{|c|c|c|}
\hline & estudiantes & grupos \\
\hline España & 30 estudiantes & curso \\
\hline Universidad & 21 locales & Diseño \\
\hline Pública de & 9 intercambio & comportamien \\
\hline Navarra & & \\
\hline & & organizacional \\
\hline Facultad & 29 estudiantes & Top $20 \%$ \\
\hline Economía $\quad \mathrm{y}$ & 22 locales & \\
\hline $\begin{array}{l}\text { Administración } \\
\text { de Empresas }\end{array}$ & 7 intercambio & \\
\hline México & 26 estudiantes & curso \\
\hline Veracruzana & & Laboratorio de \\
\hline University & & enseñanza \\
\hline Facultad & & \\
\hline Pedagogía & & \\
\hline $\begin{array}{l}\text { Total, } \\
\text { estudiantes }\end{array}$ & 85 estudiantes & \\
\hline
\end{tabular}

\section{Descripción de la plataforma utilizada}

A continuación, se describen la plataforma y las actividades realizadas por los alumnos. Moodle es una aplicación web desarrollada como una plataforma LMS (sistema de gestión del aprendizaje) en la que los estudiantes y profesores pueden generar comunidades de aprendizaje e interactuar de manera segura (mediante el registro de nombre de usuario y contraseña personal). Entre sus principales funciones, destacan facilitar la publicación de material; gestión de la comunicación a través de foros, chats y videoconferencias; y programar las actividades evaluativas. Estas características permitieron diseñar una plataforma educativa, integrando las actividades y productos que se tenían que entregar, la comunicación entre los propios estudiantes en foros, con esto cada participante ofreció sus propios puntos de vista y recibió seguimiento de los profesores.

Figura 1. Capturas de pantalla de la plataforma Moodle y las actividades de los estudiantes

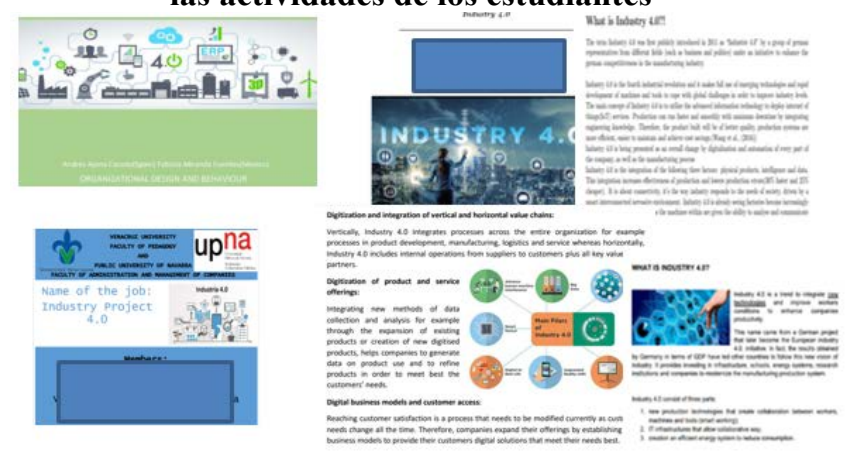

\section{Resultados}

Esta sección muestra los resultados obtenidos del análisis estadístico realizado a los estudiantes en las cuatro categorías y cada una de las puntuaciones que obtuvieron. Los datos que se arrojaron en la escala de medición fueron incluidos en Excel. La suma total por pregunta se obtuvo para cada uno de los 49 estudiantes que respondieron a dicha escala.

Los valores de escala corresponden a "1", nivel bajo, "2", nivel medio; y "3" relativo al nivel alto. Una vez obtenidos los datos, los resultados se agruparon con el fin de medir 1) qué 
competencias son las que más se desarrollaron (nivel bajo) y 2) cuáles son las que más trabajo les cuestan (nivel medio) o simplemente no pudieron llevarlas a cabo. (nivel alto).Los ejes de cada categoría son considerados y valorados con relación al logro de competencias que los alumnos desarrollaron durante el curso virtual con los compañeros con los que les toco trabajar e interactuar.

Los ejes de la Categoría I, Interacción-ComunicaciónEmpatía, Motivación y Colaboración, giraban en torno a las siguientes preguntas:

1)Capacidad para interactuar con sus compañeros

2)Capacidad de empatía

3)Capacidad motivacional para llevar a cabo actividades

4)Capacidad de Adaptabilidad

5)Capacidad colaborativa

6)Capacidad para tomar decisiones

7)Capacidad para comunicarse con sus compañeros

8) Capacidad para trabajar de manera coordinada

9)Como grupo, hemos cometido muy pocos errores sobre lo que había que hacer.

10)La comunicación entre nosotros y nosotros ha sido fluida y continua, aunque no todos estábamos presentes.

11)Creíamos que podíamos hacer un buen trabajo como grupo.

12)Hemos acordado rápidamente normas de trabajo

13) En general, hemos estado muy satisfechos con el trabajo realizado en este grupo.

14)Hemos sido un grupo eficaz.

Figura 2. Categoría I: Interacción-Comunicación-Empatía y, Motivación y Colaboración

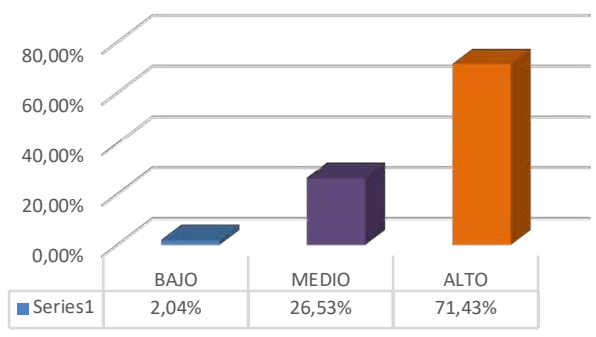

La categoría I, referida a Interacción-Comunicación, Empatía, Motivación y Colaboración, muestra que el 71,43\% de los estudiantes considera que han alcanzado un alto nivel en el desarrollo de competencias; el 26,53\% menciona haber desarrollado un nivel medio; y sólo el 2,04\% considera que alcanzó un nivel bajo (Figura 2). Esto refleja que las competencias que se promovieron en la categoría han sido favorables en el desarrollo de este curso. Podemos interpretar que se lograron comunicación, interacción, empatía, motivación, adaptación, colaboración y coordinación para la realización de las actividades para la mayoría de los estudiantes.

Los ejes de la categoría II, Dominio cognitivo-desarrollocontenido, giraban en torno a las preguntas siguientes:

1) Capacidad de comprensión de contenido (cognitiva).

2) Capacidad de síntesis de contenido.

3) Cada uno de los miembros del grupo dispone de información suficiente sobre los aspectos de la labor.
4) Tengo información sobre una parte del trabajo que ninguna otra persona en el grupo tiene.

5)Cada uno de los miembros del grupo tiene conocimientos previos suficientes para llevar a cabo la labor.

6)El conocimiento concreto de cada uno de los miembros del grupo ha sido necesario para realizar el trabajo en grupo.

7) Sé qué miembro del grupo tiene conocimiento de un aspecto específico del trabajo en grupo.

8) Me siento bien aceptando sugerencias de otras personas del grupo sobre cómo llevar a cabo la tarea grupal.

9) Confío en la información proporcionada por otras personas del grupo.

10) Tengo en cuenta y actúo de acuerdo con la información que otros miembros del Grupo contribuyen al debate del grupo.

11) Cuando otros colegas proporcionan información, necesito contrastarla o verificarla.

12) No tengo mucha confianza en el conocimiento y la información del resto de las personas del grupo

13) Hemos superado los objetivos de la labor encomendada.

Figura 3. Categoría II: Maestría en Desarrollo CognitivoContenido

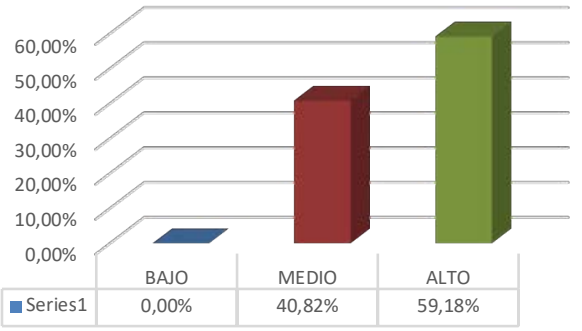

En esta categoría II, el dominio del contenido de desarrollo cognitivo se muestra que el $59,18 \%$ de los estudiantes considera haber alcanzado el desarrollo de competencias de un alto nivel, el 40,82\% menciona haber desarrollado un nivel medio y ninguno considera haber alcanzado un nivel bajo (Figura 3). Podemos ver que los estudiantes afirman que en esta categoría su desempeño fue medio-alto, por lo que tuvieron la capacidad de entender el contenido, capacidad de síntesis, gestión de la información, conocimiento de los temas por los miembros del equipo En otras palabras, lograron los objetivos de trabajo en esta categoría a nivel medio-alto.

Los ejes de la Categoría II, Uso de la búsqueda de información de la Plataforma, uso de recursos y Competencia lingüística y toma de decisiones, giraban en torno a las siguientes preguntas:

1) Capacidad para navegar por la plataforma virtual

2) Capacidad para dominar el idioma inglés

3) Capacidad para buscar información

4)Capacidad para utilizar recursos didácticos

Figura 4. Categoría III. Uso de la búsqueda de información de la plataforma, uso de recursos y competencia lingüística y toma de decisiones. 


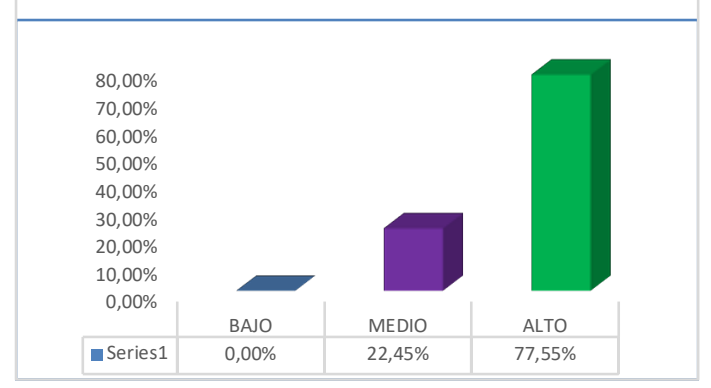

En esta categoría III, Utilice la búsqueda de información de la plataforma, el uso de recursos y el dominio del lenguaje y la toma de decisiones, se observa que el $77,55 \%$ de los estudiantes considera que han alcanzado el desarrollo de habilidades a un alto nivel, el $22,45 \%$ menciona que han desarrollado un nivel medio y ninguno considera haber alcanzado un nivel bajo (Figura 4). Podemos observar que los estudiantes afirman que en esta categoría III su desempeño fue medio-alto. Tuvieron la capacidad de navegar por la plataforma virtual, la capacidad de dominar el idioma inglés, la capacidad de buscar información y la capacidad de utilizar recursos didácticos.

Los ejes de la categoría IV, referida a la toma de decisiones, giraban en torno a las siguientes preguntas:

1) Hemos necesitado volver a los aspectos ya discutidos o rehacer algunas cosas.

2) Hemos hecho el trabajo sin problemas y eficientemente.

3) Hemos tenido suficiente confusión y dudas sobre cómo llevar a cabo el trabajo en grupo

4)Hemos acordado muy rápidamente cuáles eran nuestros objetivos

5)Hemos acordado la evaluación de nuestras habilidades y conocimientos.

6) Hemos acordado rápidamente cómo trabajar, aunque una de las personas del grupo no estaba presente.

7) Hemos abordado el caso de una manera muy similar y hemos acordado muy rápidamente el análisis de la situación

8) Inmediatamente hemos acordado lo que podríamos hacer dado el plazo asignado.

9)Cuando han aparecido diferentes puntos de vista, nos hemos escuchado, los hemos entendido y actuado en consecuencia como grupo.

10) Hemos terminado la tarea en el tiempo asignado.

11) Hemos reaccionado rápida y eficientemente cuando han surgido problemas.

12) El hecho de que los miembros del grupo no estuvieran presentes ha influido claramente en los resultados del grupo.

Figura 5. Categoría IV: Toma de decisiones

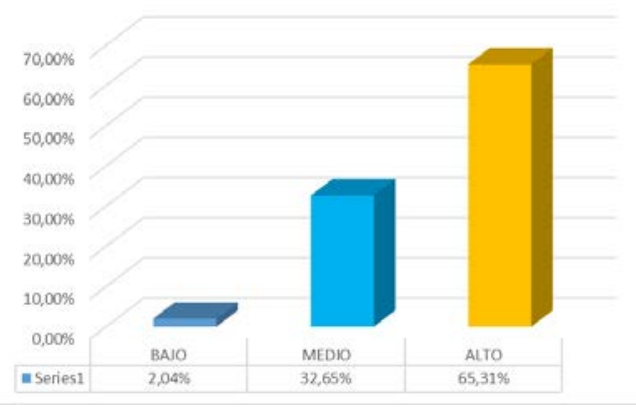

En esta categoría IV, la toma de decisiones muestra que el $65,31 \%$ de los estudiantes considera que han alcanzado el desarrollo de competencias a un alto nivel, el 32,65\% menciona que han desarrollado un nivel medio y el 2,04\% considera que han alcanzado un nivel bajo (Figura 5). En esta última sección, se observa que las competencias que se pretendían alcanzar son: haber llevado a cabo el trabajo de una manera fluida y eficiente, tener objetivos claros como equipo, haber valorado las habilidades y conocimientos del equipo, gestionar acuerdos para realizar el trabajo, tiempo de finalización de las actividades, escuchar los puntos de vista como equipo de trabajo, la entrega de las actividades en las fechas indicadas, y la capacidad de resolver los problemas que surgen, es decir, para la mayoría de los estudiantes que se lograron y sólo un porcentaje mínimo considerar no haberlos desarrollado.

Los resultados nos muestran diferencias menores. Los estudiantes expresan haber logrado el desarrollo de habilidades y habilidades digitales a un nivel medio-alto en la categoría II y la Categoría III, uso de la búsqueda de información de la plataforma, uso de recursos y competencia lingüística y toma de decisiones.

En la Categoría I, Interacción-Comunicación-Empatía, Motivación y Colaboración, y la toma de decisiones de categoría IV se pueden ver que sólo el 2,04\% de menciona no las han logrado. Sin embargo, el resto considera haber desarrollado estas habilidades a un nivel medio-alto.

Cabe mencionar, a partir de estos resultados, que las habilidades digitales son necesarias para el trabajo virtual, y permiten un mejor desarrollo para la realización de actividades, por lo tanto, nos acercan a los objetivos fijados al comienzo de la intervención.

Por último, durante la intervención tuvimos obstáculos:

- Diferencia horaria: Entre España y México hay una diferencia de 7 horas. Por lo tanto, seleccionamos los tiempos de conexión para coincidir con los horarios de ambos países.

- Idioma: Algunos estudiantes en México tienen un nivel básico intermedio en el idioma inglés, (requisito del curso) los estudiantes tuvieron que apoyar a los traductores, estudiar vocabulario y escuchar videos con el fin de entender y participar en las conversaciones.

- Formación cultural: Aunque España y México hablan la lengua española y comparten una historia común, cada uno tiene un contexto educativo, cultural, económico, político, tecnológico y educativo diferente. Cuando los alumnos se comunicaron entre sí, observamos que algunos no tenían una comprensión de algunos conceptos y/o carecían de referencias históricas.

- Diferentes áreas disciplinarias: Los estudiantes de España eran del área de economía empresarial; los de México estudiaban humanidades, ambos están familiarizados con conceptos y enfoques diferentes, sin embargo, el tema de la tecnología es global e implica que los estudiantes desarrollen habilidades que les permitan incorporarlos en el lugar de trabajo. Podemos decir que los estudiantes aprendieron de ambas áreas y cada uno lo aplica a su contexto disciplinario.

- La conexión remota por videoconferencia era lenta y requería volver a conectarnos constantemente.

\section{CONCLUSIONES}

Ratten y Jones (2020a; 2020b) destacaron la relevancia de introducir más investigación educativa sobre la transformación 
digital y el entorno tecnológico en la educación de gestión. Este documento tiene como objetivo responder a esta convocatoria. Por otro lado, Echeverría (2000) afirmó que el contexto virtual implica una nueva identidad (E3, identidad digital) que coexiste con la identidad genérica (E1) y la identidad social (E2). Los individuos deben actuar en las tres dimensiones. La OCDE (2019) refuerza esta afirmación reconociendo que, para prosperar en el lugar de trabajo digital, no sólo las competencias digitales, sino también las cognitivas y socioemocionales. La investigación actual contribuye a esta literatura elaborando esta idea en un experimento internacional. Según nuestros resultados, las cuatro categorías alcanzaron un nivel medio-alto, es decir, fueron desarrolladas por los estudiantes en un curso basado en la Industria 4.0 y la transformación digital.

Sobre la base de los datos recogidos y analizados y, también, las percepciones de los participantes, se puede mencionar que la mayoría expresó una posición favorable sobre el impacto del curso. Esto fue seguido por un impacto neutral. Menos del 5\% de los estudiantes mencionaron un impacto negativo. Por lo tanto, se puede concluir que se alcanzaron los objetivos del estudio.

Respecto a los resultados principales del estudio, nos permitió conocer las competencias y habilidades que los estudiantes desarrollaron al máximo, y cuáles necesitaban mejorar. Al mismo tiempo, las actividades dinámicas y didácticas contribuyeron al logro de las actividades, como se reflejó en los comentarios de los estudiantes. La comunicación remota entre los estudiantes permitió trabajar de manera consensuada y colaborativa. Los estudiantes estuvieron de acuerdo en su estilo de conectar entre sí para llevar a cabo las actividades (en horas tardías), lo que implicaba responsabilidad y toma de decisiones para lograr el objetivo y subir sus comentarios y trabajo en los tiempos establecidos.

En un tercer aspecto, interactuar con estudiantes de otro país y otras áreas académicas permitió a los estudiantes tener una visión diferente de los conceptos y temas, y cómo se aplican en cada uno de los diversos contextos. Destacar que el seguimiento en los foros permitió a los estudiantes expresar sus puntos de vista, analizar, criticar, comparar y reflexionar sobre cada uno de los temas.

En cuanto a la investigación futura, es importante mencionar que el aula virtual es un espacio donde no sólo se transmite el conocimiento, sino también donde se comparten experiencias. Se desarrollan habilidades de comunicación, interactivas, colaborativas, motivacionales y de toma de decisiones; se fomenta la empatía y la adaptabilidad; y la capacidad de intercambiar ideas en un idioma distinto al suyo. Por ello, es importante generar actividades que permitan al estudiante desarrollar dichas competencias, que se apliquen en su vida diaria y desarrollo profesional.

En resumen, esta intervención busca promover nuevos avances en este ámbito. De este modo, se pueden aplicar cursos similares de esta naturaleza como medio para promover el aprendizaje virtual y el desarrollo de habilidades digitales en los estudiantes, creando así espacios virtuales positivos para el aprendizaje.

\section{REFERENCIAS}

Castells, M. (2010). The Information Age. Economy, Society and Culture. The Rise of the Network Society. Chichester: Wiley-Blackwell.
De Castro, Cristina. (2016). Acquiring autonomy at school and in work group through information and communication technologies. Conference paper presented at the International Technology, Education and Development

Fandos M. (2003). Training based on Information and Communication Technologies. Didactic analysis of the teaching-learning process. Tarragona.

Goldhammer, F., Gniewosz, G., \& Zylka, J. (2016). ICT engagement in learning environments. In Assessing Contexts of Learning (pp. 331-351). Springer, Cham. https://doi.org/10.1007/978-3-319-45357-6_13

Hernández R, Fernández C, Baptista P. (2010). Investigation methodology. Federal District, Mexico. Ed. Mc. Graw Hill.

Martinovic, D., Freiman, V., Lekule, C. S., \& Yang, Y. (2019). The Roles of Digital Literacy in Social Life of Youth. In Advanced Methodologies and Technologies in Library Science, Information Management, and Scholarly Inquiry, 103-117. IGI Global. https://doi.org/10.4018/978-1-5225-7659-4.ch009

Mishna, F., Regehr, C., Lacombe-Duncan, A., Daciuk, J., Fearing, G., \& Van Wert, M. (2018). Social media, cyberaggression and student mental health on a university campus. Journal of mental health, 27(3), 222-229. https://doi.org/10.1080/09638237.2018.1437607

OECD (2017). OECD Skills Strategy Diagnostic Report. OECD Skills Studies, OECD Publishing, Paris.

OECD (2019). OECD Skills Strategy 2019: Skills to shape a better future. OECD Publishing, Paris.

Picatoste, J., Pérez-Ortiz, L., Ruesga-Benito, S. M., \& NovoCorti, I. (2018). Smart cities for wellbeing: youth employment and their skills on computers. Journal of Science and Technology Policy Management, 9(2), 227241. https://doi.org/10.1108/JSTPM-04-2017-0014

Ratten, V., \& Jones, P. (2020a). Entrepreneurship and management education: Exploring trends and gaps. The International Journal of Management Education, 100431. In-press. https://doi.org/10.1016/j.ijme.2020.100431

Ratten, V., \& Jones, P. (2020b). Covid-19 and entrepreneurship education: Implications for advancing research and practice. The International Journal of Management Education, $100432 . \quad$ In-press. https://doi.org/10.1016/j.ijme.2020.100432

Rueda J, (2007). Technology in 21 st centrury society. Dawn of a new Industrial revolución. Taken from the Journal of Social Sciences, No. 32, January-March 2007. Móstoles, Spain. Available in: https://www.redalyc.org/articulo.oa? $\mathrm{id}=495950225001$. Accessed. Accessed 18 January, 2020.

Wang, H. Y., Sigerson, L., Jiang, H., \& Cheng, C. (2018). Psychometric Properties and Factor Structures of Chinese Smartphone Addiction Inventory: Test of Two Models. Frontiers in psychology, 9, 1411. https://doi.org/10.3389/fpsyg.2018.01 